\title{
Robust Copyright Protection of Satellite Images Using a Novel Digital Image-In-Image Watermarking Algorithm
}

\author{
Anthony Tung Shuen Ho ${ }^{*}$ \\ Nanyang Technological University, School of Electrical and Electronic Engineering \\ Nanyang Avenue, Singapore 639798
}

Tel: (65) 7906373 Fax: (65) 7937790 Email: etsho@ntu.edu.sg

* Co-founder and Director of DataMark Technologies

www.datamark-tech.com

\begin{abstract}
In this paper, a novel digital watermarking technique for copyright protection of satellite images is presented. This robust technique can survive a number of image manipulations or "attacks" such as contrast stretching, rotation, clipping and filtering to the watermarked image. Conventional digital watermarking techniques use a number of bits or characters to be embedded into the image. However, many of these techniques would yield to some minor attacks to the watermarked image due to the alteration of the values of these bits or characters. As a result, the legal ownership of the data under copyright protection would be questionable. The performance of any digital watermarking algorithms depends on the tradeoff between data integrity and the robustness of the algorithms.
\end{abstract}

Keywords: Digital Watermarking, Steganography, Copyright Protection, Satellite Images

\section{INTRODUCTION}

With the advent of the Internet, the purchasing and distribution of satellite images can be now performed online with relative ease. With this ease come the problem of copyright protection and the proof of rightful ownership of satellite images. The prevention of illegal distribution of digital multimedia content on the Internet and other media has led to recent interest and research into the field of digital watermarking $[1,2]$ and digital rights management (DRM).

This paper presents a novel digital watermarking algorithm using an image as a watermark to be hidden in an original image. Satellite images with various complexities will be used to illustrate the robustness of the algorithm against various image attacks. Although some artifacts can be visualized from the retrieved watermark after the attacks, the overall structure of the watermark remained intact and is still recognizable. This paper will provide different watermarking attack examples on satellite images and discusses the performance tradeoffs in terms of data integrity and algorithm robustness.
Digital watermarking is essentially a subset of steganography. Steganography is a Greek word meaning "hidden writing" or "covered writing". Many digital watermarking techniques adopt similar technologies used in transform coding and communication theory [2]. However, many of these techniques can only embed or hide a limited number of bits or characters in the original data. In this paper, a novel image-in-image watermarking technique known as StegMark developed by DataMark Technologies [3] was applied to various satellite images. StegMark allows an image watermark, such as a company's logo to be embedded inside the satellite images, while maintaining the data integrity and robustness of the watermark.

Figure 1 illustrates how StegMark performs the digital watermarking process. Once the watermark, w, has been embedded inside the original image, a hash or key file is created. The hash file is used to retrieve the hidden watermark and will be distributed to and kept by the rightful owner. It will be used in a legal dispute to identify the true ownership of the data.

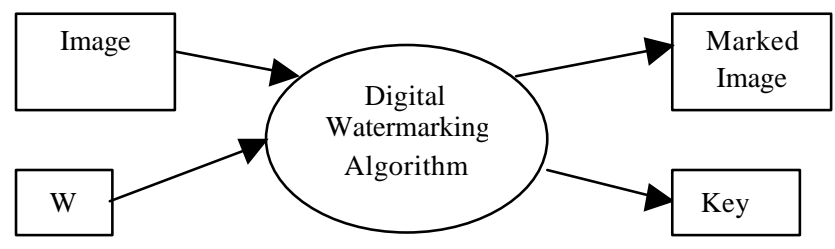

Figure 1 illustrates the digital watermarking process

Using StegMark, three satellite images with various complexities, taken from SPOT and Landsat of pixel size $512 \times 512$ were embedded with the same image watermarks. Figure 2 illustrates the original and watermarked images. An image watermark with the character "DMT" of pixel size $100 \times 40$ was used for the embedding. The PSNR of the three watermarked images were $89.82 \mathrm{~dB}$ and $84.79 \mathrm{~dB}$ for the SPOT Hongkong and Sea images and $94.38 \mathrm{~dB}$ for the Landsat image, respectively. 


\section{WATERMARK ROBUSTNESS}

There are many different watermarking techniques commercially available and from various research institutions [1] based on bit and character embedding. However, many of these algorithms can be easily attacked and have their watermarks removed from the original image. Image attacks such as contrast stretching, flipping, format conversion can be used relatively easily to remove the watermark. To measure the effectiveness of a watermarking technique in terms of its robustness and data integrity criteria, an image manipulation tool called Stirmark is commonly used to perform the performance benchmarking [1]. Stirmark consists of around 90 different types of image attacks that include rotation, scaling and filtering. Figure 3 demonstrates the robustness of StegMark against the various attacks such as $20 \%$ cropping, rotation by $0.25^{\circ}$ and JPEG compression with a factor of 50 .

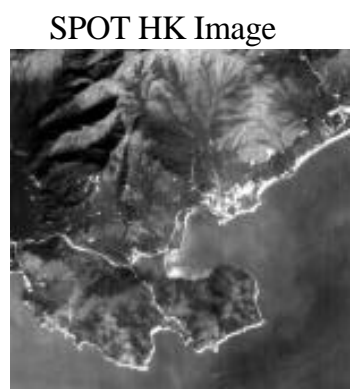

Landsat Image

SPOT Sea Image

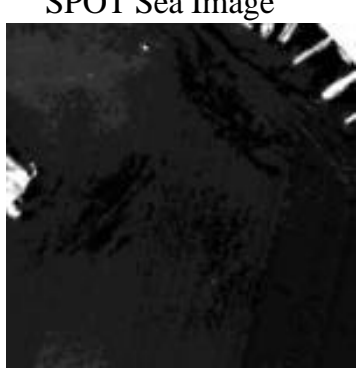

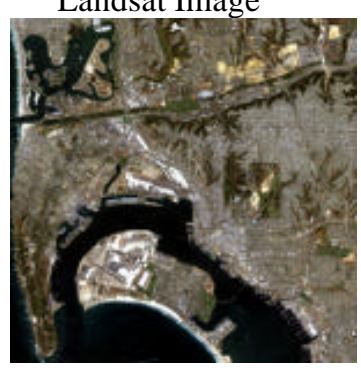

SPOT HK Watermarked

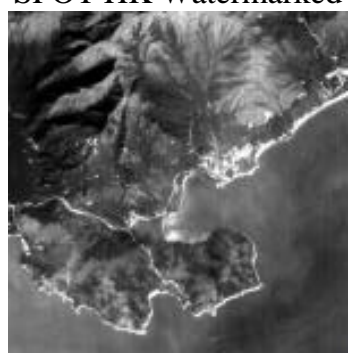

Landsat Watermarked
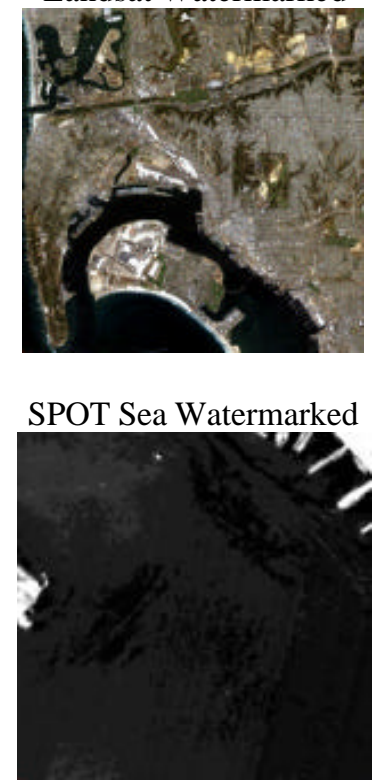

Figure 2 illustrates the original and watermarked satellite images of various complexities.
From figure 3, the image watermark with characters "DMT" clearly survived various attacks from Stirmark. Although, the recovered watermarks were contaminated with various artifacts, however; the main advantage of the StegMark watermarking technique is its ability to exploit our own powerful visual system (our eyes) to recognize the structure of the images. Conventional watermarking techniques typically use some form of confidence level based on a pre-determined threshold to check whether the watermark characters or bits are the same after image attacks. The results basically provide either a "yes" or "no" answer.

For these techniques using bits or characters-based, the binary 1 or 0 can be modified quite easily to become 0 or 1 under attacks. For example, an ID string "ABC" as a watermark can be retrieved as "BBC" after certain type of attacks. Character "A" can be represented in ASCII by a decimal value 65 and " $\mathrm{B}$ " by 66 . The decimal values are only different by 1 . The question remains on the robustness of watermarking algorithms under various forms of image manipulations. Using the Stirmark benchmark test, the novel StegMark watermarking algorithm achieved a success rate of approximately $89 \%$ for the 90 different image attacks.

\section{CONCLUSIONS AND FUTURE WORK}

This paper described a novel image-in-image watermarking algorithm for copyright protection of satellite images. Two SPOT and one Landsat images with various complexities were used for the investigation. Using Stirmark, which is a standard benchmarking tool to attack watermarks, the StegMark algorithm was able to achieve close to $89 \%$ success rate against more than 90 image manipulations or attacks that included format conversion, rotation, contrast stretching, scaling and filtering. The PSNR was used to compare the original and the watermarked satellite images and the results ranged from approximately $85 \mathrm{~dB}$ to $95 \mathrm{~dB}$.

As the watermark is a 2-D image, it is planned that some post-processing such as image restoration and correlation can be used to further enhance the watermark recognition process and therefore increase the success rate against different types of image attacks. Another area that we are currently investigating is the ability to perform digital watermarking for printed materials. For example, if the satellite image has been developed as a photograph or printed, can we still retrieve the watermark? 


\section{REFERENCES}

1. Katzenbeisser, S, Petitcolas. F, "Information Hiding Techniques for Steganography and Digital Watermarking", Published by Artech House, Computer Security Series, 2000

2. Cox, I, Kilian, J, Leighton, T, Shamoon, T, "Secure Spread Spectrum Watermarking for Multimedia," IEEE Trans on Image Processing, Vol 6, 12, Dec 1997

3. Ho, A.T.S. et.al., "Digital steganography for Information Security", Internet Business99, CommunicAsia99, 22-25 June 1999, Singapore

Figure 3 illustrates the various image attacks on the watermarked satellite image and the recovered watermark

Original watermarked image $\quad 20 \%$ cropping

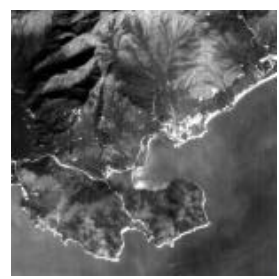

DMT
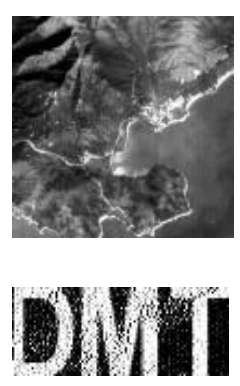

Rotation clockwise $0.25^{\circ}$
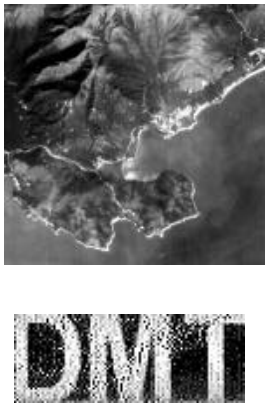

JPEG compression with factor 50
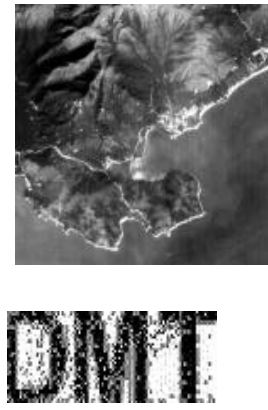

Table 1. Image Attacks and PSNR of Watermarked Satellite Image and Extracted Watermarks (shaded ones indic ated failed test)

\begin{tabular}{|c|c|c|}
\hline Attacks & $\begin{array}{c}\text { Image PSNR(dB) } \\
\text { under attacks }\end{array}$ & $\begin{array}{l}\text { Extracted watermark } \\
\text { PSNR(dB) }\end{array}$ \\
\hline Cropping 20\% & NA & 12.01 \\
\hline 1 row 5 columns removed & $\overline{\mathrm{NA}}$ & 31.48 \\
\hline 5 rows 1 column removed & NA & 17.42 \\
\hline $2 \times 2$ Median Filtering & 26.69 & 17.99 \\
\hline $4 \times 4$ Median Filtering & 23.45 & 28.18 \\
\hline Frequency Mode Laplacian removal & 31.26 & 26.43 \\
\hline Gaussian filtering $3 \times 3$ & 31.05 & 22.96 \\
\hline JPEG Compression of factor 50 & 32.32 & 31.69 \\
\hline JPEG Compression of factor 30 & 31.65 & 23.29 \\
\hline JPEG Compression of factor 15 & 29.09 & 22.22 \\
\hline $\begin{array}{l}\text { General linear transformation } \\
{\left[\begin{array}{lll}1.007 & 0.010\end{array}\left[\begin{array}{ll}0.010 & 1.012\end{array}\right]\right.}\end{array}$ & NA & 19.31 \\
\hline Scaling 1.1 & NA & 18.13 \\
\hline Change aspect ratio $\mathrm{X}-1.2 \mathrm{Y}-1.0$ & $\overline{\mathrm{NA}}$ & 34.87 \\
\hline Change aspect ratio $\mathrm{X}-1.0 \mathrm{Y}-1.2$ & NA & 19.71 \\
\hline Rotation $-2^{\circ}$ with cropping & NA & 19.87 \\
\hline Rotation $-1^{\circ}$ with cropping and scaling & 18.45 & 20.10 \\
\hline Sharpening $3 \times 3$ & 19.28 & 54.52 \\
\hline Shearing $\mathrm{X}-1 \% \quad \mathrm{Y}-1 \%$ & NA & 18.13 \\
\hline Stirmark with randomisation and bending & 16.80 & 20.03 \\
\hline
\end{tabular}

